\title{
A synthetic carbohydrate conjugate vaccine candidate against shigellosis: improved bioconjugation and impact of alum on immunogenicity
}

\author{
Robert M. F. van der Put, ${ }^{\mathrm{a}, \downarrow}$ Tae Hee Kim,,${ }^{\mathrm{b}, \mathrm{c}, \downarrow}{ }^{\dagger}$ Catherine Guerreiro, ${ }^{\mathrm{b}, \mathrm{c}}$ Françoise Thouron, ${ }^{\mathrm{d}, \mathrm{e}}$ \\ Peter Hoogerhout, ${ }^{\text {a }}$ Philippe J. Sansonetti, ${ }^{\text {d,e }}$ Janny Westdijk, ${ }^{\text {a }}$ Michiel Stork, ${ }^{a}$ Armelle \\ Phalipon, ${ }^{\mathrm{d}, \mathrm{e}}$ Laurence A. Mulard ${ }^{\mathrm{b}, \mathrm{c}^{*}}$ \\ ${ }^{\mathrm{a}}$ Institute for Translational Vaccinology (Intravacc), P.O. Box 450, 3720 AL Bilthoven, the \\ Netherlands \\ ${ }^{\mathrm{b}}$ Institut Pasteur, Unité de Chimie des Biomolécules, 28 rue du Dr Roux, 75724 Paris Cedex \\ 15, France \\ ${ }^{\mathrm{c}}$ CNRS UMR 3523, Institut Pasteur, F-75015 Paris, France \\ ${ }^{\mathrm{d}}$ Institut Pasteur, Unité de Pathogénie Microbienne Moléculaire, 28 rue du Dr Roux, 75724 \\ Paris Cedex 15, France \\ e INSERM U1202, Institut Pasteur, F75015 Paris, France \\ * Corresponding author's e-mail: laurence.mulard@pasteur.fr
}

\section{Table of content}

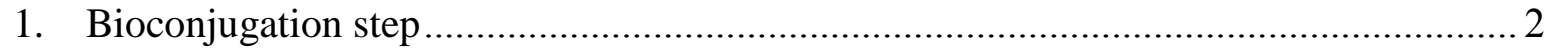

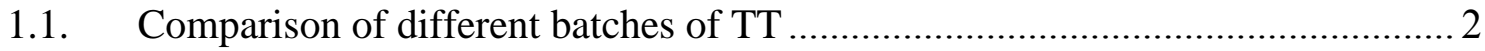

1.2. Influence of buffers and organic solvents on TT to intermediate 4 conversion... 2

1.2.1. Comparing different buffers for the grafting of GMBS on TT .......................... 2

1.2.2. Aggregation induction of different organic solvents .......................................... 3

1.2.3. Characterization of TT activation ................................................................. 4

1.3. Design of Experiments (DoE) and analysis ................................................... 9

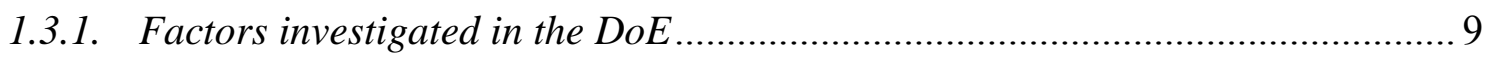

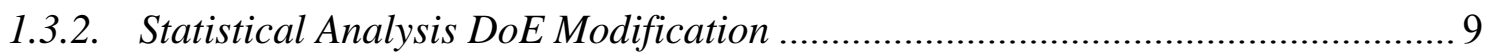

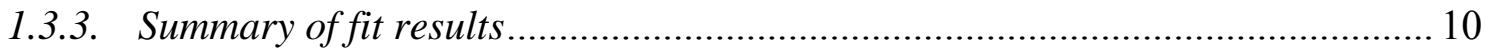

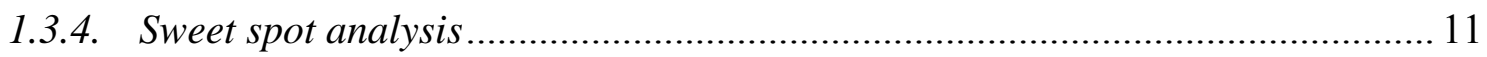

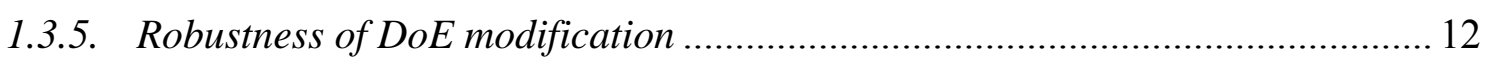

1.3.6. Removal of free pentadecasaccharide after conjugation .................................. 13

2. Immunogenicity analysis .............................................. 14 


\section{Bioconjugation step}

\subsection{Comparison of different batches of TT}

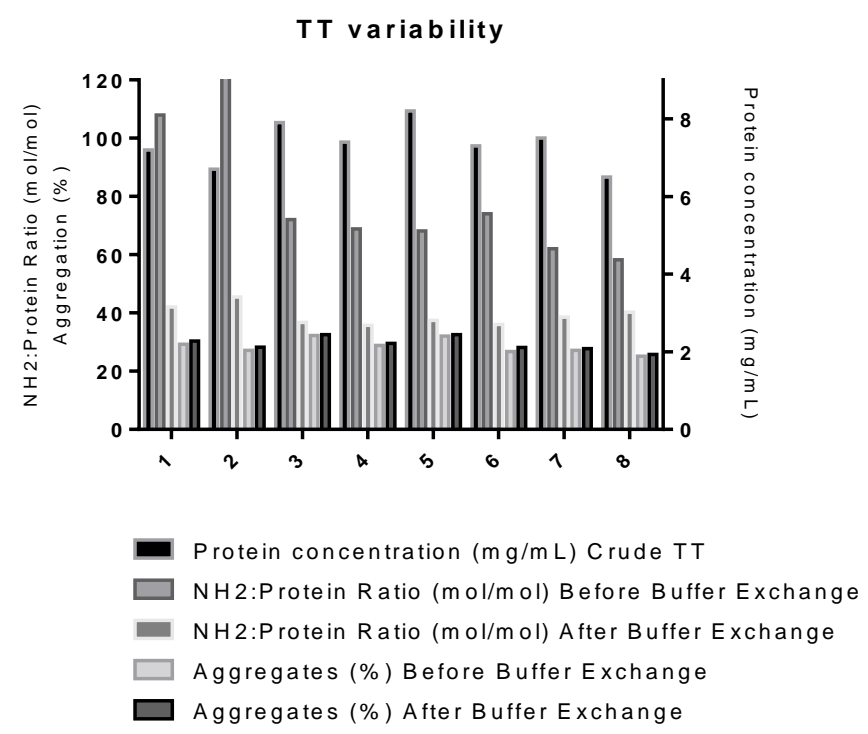

Figure S1: Comparison of different batches of TT before and after buffer exchange. Batch 3 was used for all other experiments due to availability.

\subsection{Influence of buffers and organic solvents on $\mathrm{TT}$ to intermediate $\mathbf{4}$ conversion}

\subsubsection{Comparing different buffers for the grafting of GMBS on TT}

NMR analysis showed that both GMBS and sulfo-GMBS hydrolyzed at $\mathrm{pH} 7$ and 8 in $0.1 \mathrm{M}$ phosphate buffer. Next to the reaction of the NHS ester, a reaction of the maleimide group was observed. Considering the degradation of the NHS-ester, a $T^{1 / 2}$ of 80 and 50 min was observed for GMBS at pH 7 and 8, respectively. For sulfo-GMBS a $\mathrm{T}^{1 / 2}$ of $30 \mathrm{~min}$ at $\mathrm{pH} 7$ and a $\mathrm{T}^{1 / 2}$ of $75 \mathrm{~min}$ at $\mathrm{pH} 8$ was observed. NMR analysis of both reagents dissolved in $0.1 \mathrm{M}$ HEPES at the here fore mentioned $\mathrm{pHs}$, showed an increase of the $\mathrm{T}^{1 / 2}$ to multiple hours, or even days (data not shown).

In the following modification experiments, only GMBS was used because it was dissolved in DMSO, and found to be stable for prolonged time (days), whereas sulfo-GMBS was dissolved in the aqueous buffer, and would start to degrade immediately even in 0.1 M HEPES. Four different ratios were compared at two different time points (15 and $60 \mathrm{~min}$ ) for both phosphate and HEPES buffer (Table S1).

When evaluating the HPSEC profiles, where signals were normalized for the TT peak corresponding to monomeric TT, it was concluded that the modification in $0.1 \mathrm{M}$ phosphate buffer $\mathrm{pH} 8$ induces significantly higher quantities of aggregates (45-55\%) than modification 
in 0.1 M HEPES buffer pH 7.5 (35-45\%) (data not shown). The reaction efficiency itself was very similar for both buffers, as was the result after 15 and 60 min for both primary amine group quantity (data not shown) and aggregate content (Figure $\mathrm{S} 2, \mathrm{t}=15 \mathrm{~min}$ ). These results in combination with the prolonged stability justified the selection of a 0.1 M HEPES buffer and the use of only GMBS for further experiments. Furthermore, DMSO was the solvent of choice due to its European Pharmacopoeia (EP) grade availability, which was beneficial for future GMP production activities.

Table S1: Modification parameters for GMBS using different buffers

\begin{tabular}{|c|c|c|c|c|c|c|c|c|}
\hline & \multicolumn{4}{|c|}{ In 0.1M Phosphate pH 8 } & \multicolumn{3}{|c|}{ In 0.1M HEPES pH 7.5 } \\
\hline TT $^{\text {a }}$ GMBS ratio (mol:mol) & 150 & 80 & 40 & 20 & 150 & 80 & 40 & 20 \\
\hline GMBS amount $(\mu \mathrm{mol})$ & 10.5 & 5 & 2.8 & 1.4 & 10.5 & 5.6 & 2.8 & 1.4 \\
\hline
\end{tabular}

a: TT: $300 \mu \mathrm{L}, 35 \mathrm{mg} / \mathrm{mL}, 70 \mathrm{nmol}$; GMBS solution: $0.5 \mathrm{M}, 20 \mu \mathrm{L}$

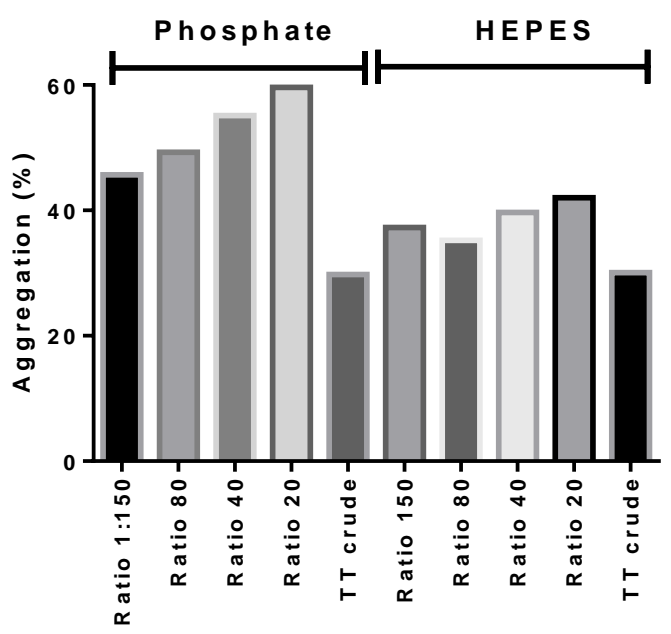

Figure S2: Influence of the buffer on TT aggregation after 15 minutes reaction time. The ratio described for each sample is the GMBS:TT ratio.

\subsubsection{Aggregation induction of different organic solvents}

Different organic solvents (DMSO, acetonitrile and DMA) were added to TT to investigate aggregation induction. TT was concentrated and buffer exchanged to either $0.1 \mathrm{M}$ phosphate pH 8.0 or 0.1 M HEPES pH 7.5 before adding the organic solvents. When adding $3.5 \mu \mathrm{L}$ of the respective organic solvents (highest amount of solvent use during experiments) to $50 \mu \mathrm{L}$ 
olvents

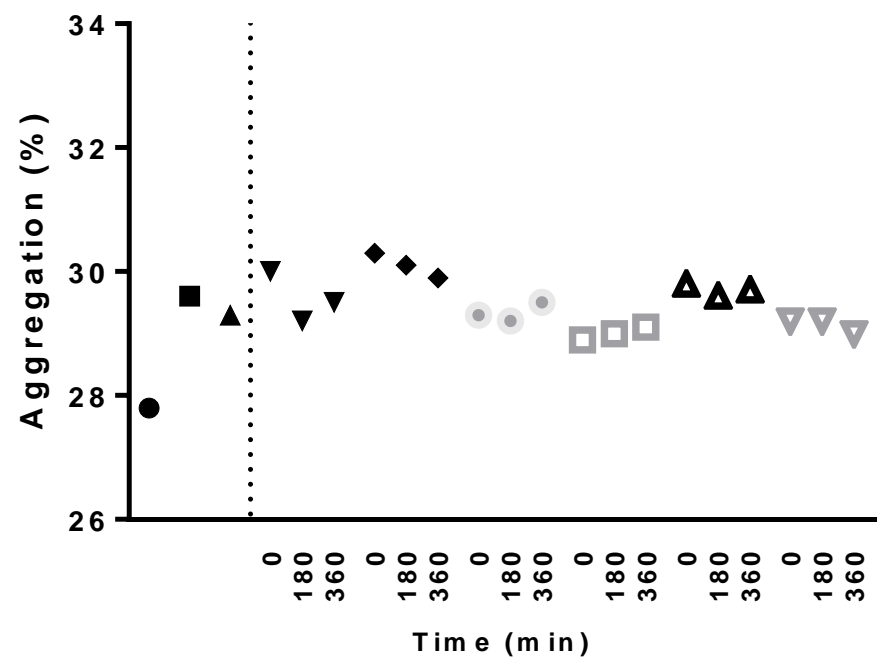

- TT Crude

- TT Phosphate

- Tt hepes

$\checkmark$ Phosphate + DMSO

- HEPES + DMSO

- Phosphate + DMA

口 HEPES + DMA

$\triangle$ Phosphate + acetonitrile

$\nabla$ HEPES + acetonitrile

Figure S3: Influence on the aggregation of TT when adding an organic solvent. TT crude: unprocessed TT without both buffer exchange and added solvents; TT Phosphate: buffer exchanged TT without added solvents; TT HEPES: buffer exchanged TT without added solvents

\subsubsection{Characterization of TT activation}

Within this investigation, the final volume of the buffer-exchanged concentrated TT was 3.2 $\mathrm{mL}$, with a final concentration factor of 10 . From this concentrate, $3.0 \mathrm{~mL}$ was applied on a $11 \mathrm{~mm}$ Millipore Vantage L Biochromatography laboratory column $(89 \mathrm{~cm})$ with a Sepharose 6FF matrix (GE Lifesciences, $84.55 \mathrm{~mL}$, Plates 1431, Asymmetry 1.45). The applied flow rate was $0.5 \mathrm{~mL} / \mathrm{min}$ and fraction size was $2.0 \mathrm{~mL}$. A $2 \mathrm{~mm} \mathrm{UV}$ cell was used for detection, coupled to an AKTA system (GE-Life sciences). The Unicorn software (GE Lifesciences, V 5.11) was used for data acquisition and processing.

It was decided to pool several designated fractions to create a pool of interest to be used for the modification experiments. In total four different pools of interest could be assigned (Table S2). Pool one is considered to contain high molecular weight oligomers (fractions $1-3$ ), pool two to contain intermediate oligomers (fractions $4-8$ ), pool three to contain dimers of TT 
(fractions 9 - 15) and pool four to contain TT mostly in its monomeric form (fractions 18 22). After pooling the fractions, HPSEC analysis confirmed the assumed composition (Figure S4).

Table S2: SEC fraction pools in 0.1M HEPES pH 7.5

\begin{tabular}{|c|c|c|c|c|}
\hline Pool & $\begin{array}{c}\text { Fractions } \\
(\mathbf{2 . 0} \mathbf{~ m L} \text { each })\end{array}$ & $\begin{array}{c}\text { BCA } \\
(\mathbf{m g} / \mathbf{m L})\end{array}$ & $\begin{array}{c}\text { TNBS } \\
(\mathbf{m M})\end{array}$ & $\begin{array}{c}\text { Ratio (NH2:TT } \\
\mathbf{m o l}: \mathbf{m o l})\end{array}$ \\
\hline 1 & $1-3$ & 1.538 & 0.465 & 45.4 \\
\hline 2 & $4-8$ & 0.823 & 0.167 & 30.4 \\
\hline 3 & $9-15$ & 8.953 & 1.649 & 27.6 \\
\hline 4 & $18-22$ & 30.403 & 6.178 & 30.5 \\
\hline
\end{tabular}

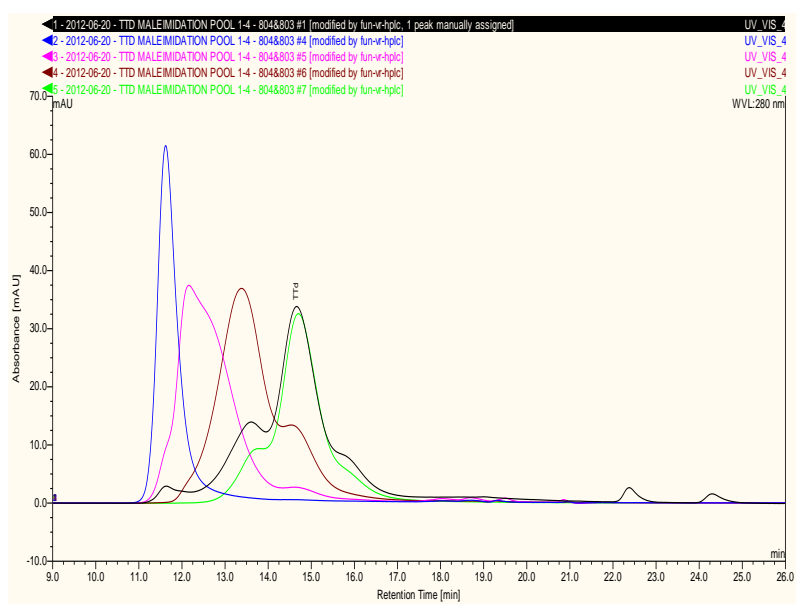

Figure S4: HPSEC profile of TT and pooled fractions. Blue: pool 1, Pink: pool 2, Brown: pool 3, Green: pool 4 and Black: TT. x-axis: retention time (min), y-axis left $\mathrm{UV}_{280} \mathrm{mAU}$

All individual pools were modified by applying different GMBS to TT ratios $(20,40,80$ and 150, respectively). The reaction time was 15 minutes at ambient temperature, after which buffer exchange was initiated. When evaluating the HPSEC profiles (signals normalized on peak height, but not retention time), it could be concluded that for pool 1 no difference could be observed between the pool and the modified oligomers (Figure S5). For pools 2 and 3, only a minor shift in retention time is observed, which could suggest an effect of the GMBSactivation reaction (Figures S6 and S7). For pool 4, a minor shift in retention time is also observed. Again, it can be interpreted as a result of the covalent attachment of maleimide groups. In addition, the induction of aggregates for pool 4 is significant (Figure S8). An 
inverse proportional relationship exists between the GMBS:protein ratio and the induction of aggregation (the higher the ratio the lower the induction of aggregation).

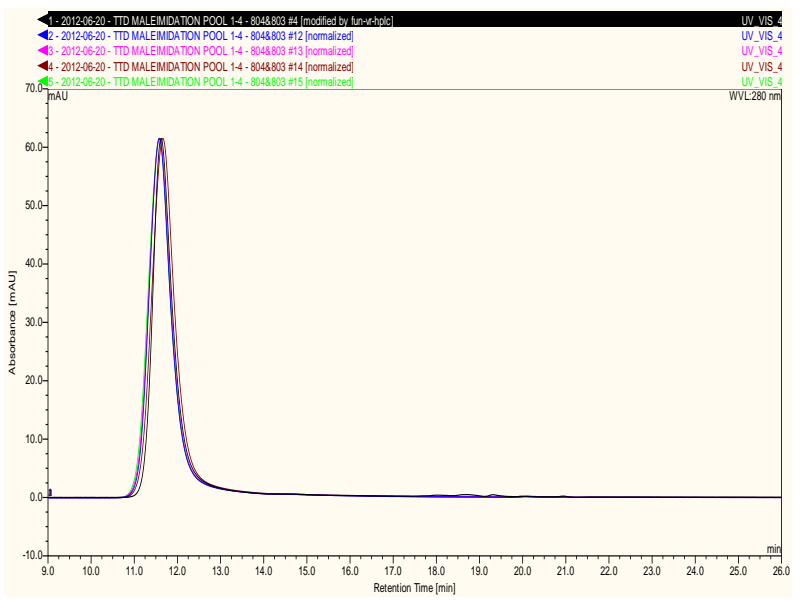

Figure S5: Pool 1 before and after treatment with GMBS at different GMBS:protein ratios, $x-$ axis: retention time (min) y-axis left $\mathrm{UV}_{280} \mathrm{mAU}$

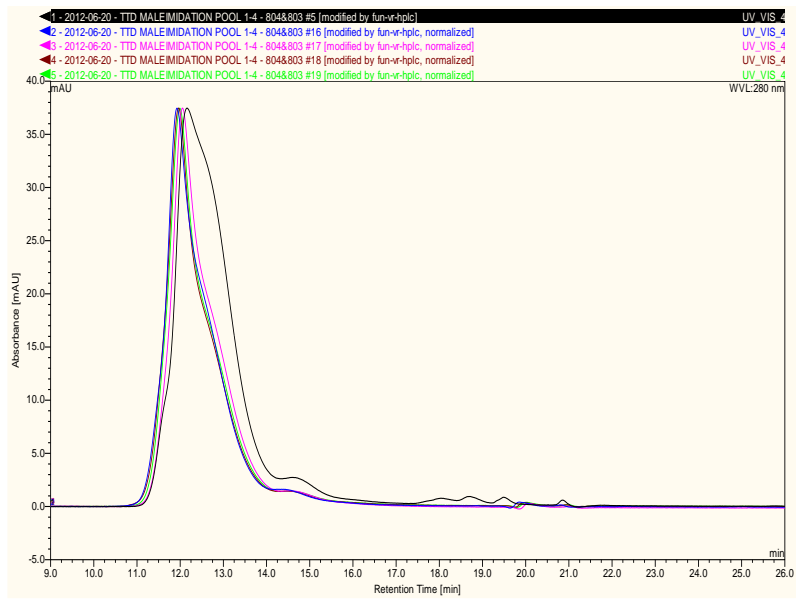

Figure S6: Pool 2 before and after treatment with GMBS at different GMBS:protein ratios, $x-$ axis: retention time (min), mAU y-axis left $\mathrm{UV}_{280} \mathrm{mAU}$ 


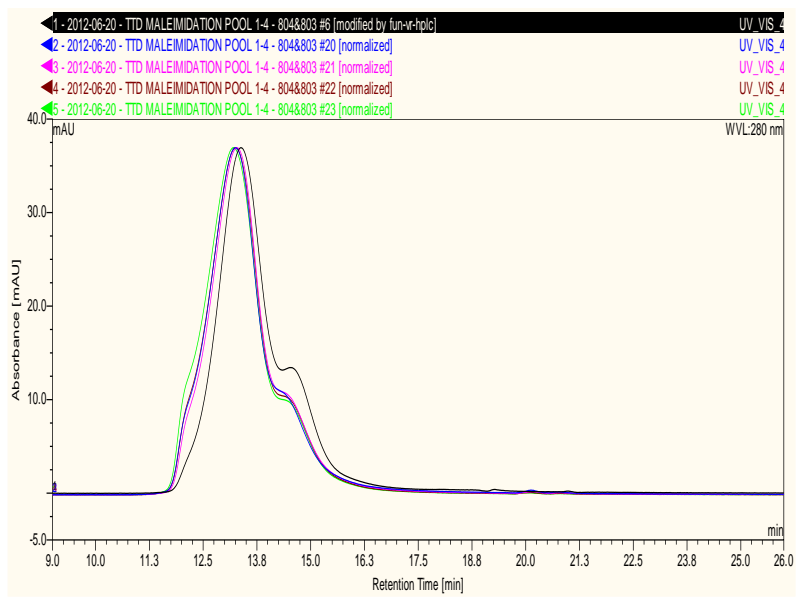

Figure S7: Pool 3 before and after treatment with GMBS at different GMBS:protein ratios, $\mathrm{x}-$ axis: retention time (min), y-axis left $\mathrm{UV}_{280} \mathrm{mAU}$

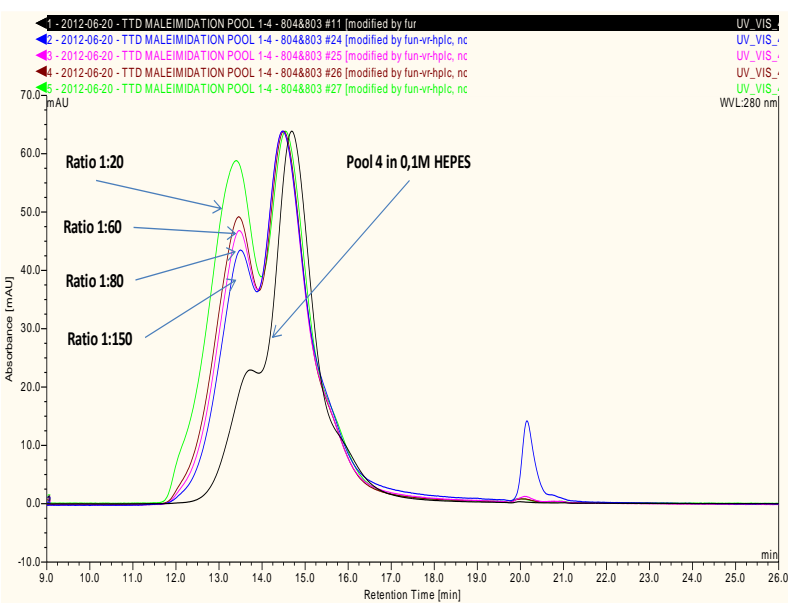

Figure S8: Pool 4 before and after treatment with GMBS at different GMBS:protein ratios, $\mathrm{x}-$ axis: retention time (min), $y$-axis left $\mathrm{UV}_{280} \mathrm{mAU}$

The initial quantity of primary amino groups (Figure S9) for pool 1 is significantly different from that of the other pools. This difference remains (although less pronounced) after the GMBS-modification reaction. For all pools, similar dynamics apply; the higher the GMBS:protein ratio the higher the modification ratio (reduction in the amount of primary amino groups per TT).

When multiplying the average number of $\mathrm{NH}_{2}$ groups per protein by the average protein concentration of the original pools, the total contribution of each individual pool is better visualized (Figure S10). Here, it becomes apparent that even though pool 1 has a higher $\mathrm{NH}_{2}$ mol:mol content, the contribution is marginal when corrected. Pool 4, which is the monomeric TT, has the largest contribution. As a result from this experiment it was decided that the 
available original TT batch, with its substantial amount of oligomers, was useable for further process development, since the larger oligomers only contribute marginally to the total amount of reactive $\mathrm{NH}_{2}$ groups ( $2 \%$ for pool 1), and therefore to the GMBS-modification reaction. On the other hand, aggregation of monomeric TT, induced by the modification, needs to be minimized.

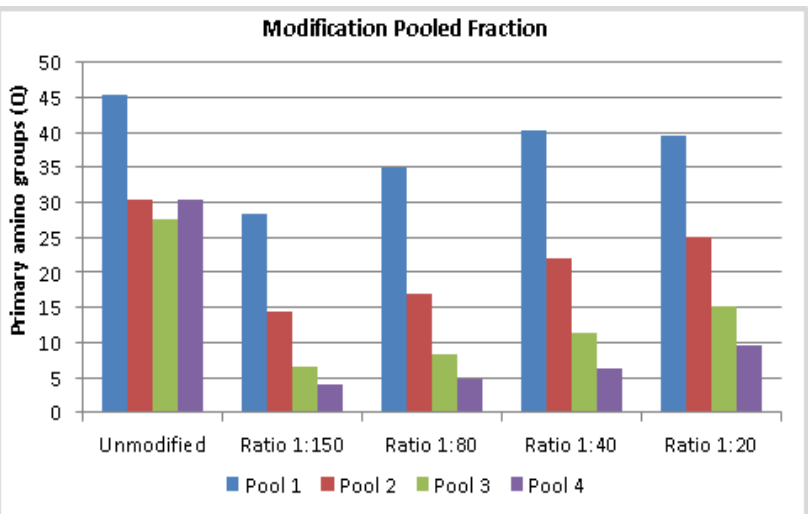

Figure S9: Primary amino group quantities for the pools and the GMBS-modified products

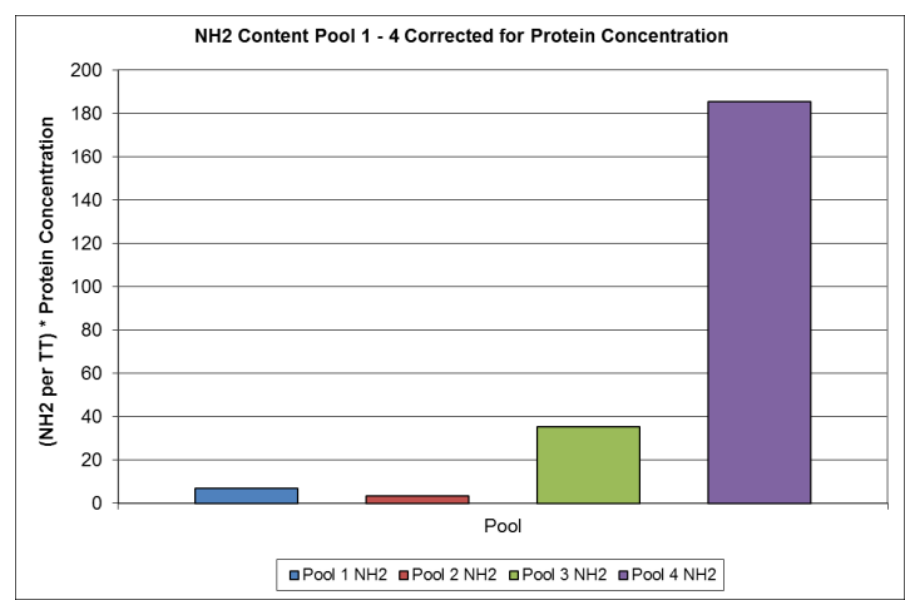

Figure S10: Corrected primary amino group quantities for the pools of the GMBS-modified modified products 
1.3. Design of Experiments (DoE) and analysis

\subsubsection{Factors investigated in the DoE}

In order to find the optimal conditions for introduction of the maleimide linker, several factors were investigated using DoE. A full overview of all the different combinations of factors is given below (Table S3).

Table S3: DoE reaction conditions

\begin{tabular}{|c|c|c|c|c|c|c|c|}
\hline $\begin{array}{c}\text { Exp } \\
\mathbf{N b}\end{array}$ & $\mathbf{p H}$ & $\begin{array}{c}\text { Temperature } \\
\left({ }^{\circ} \mathbf{C}\right)\end{array}$ & $\begin{array}{c}\text { TT } \\
\text { concentration } \\
(\mathbf{m g} / \mathbf{m L})^{\mathbf{a}}\end{array}$ & $\begin{array}{c}\text { TT } \\
\text { amount } \\
(\mathbf{m g})\end{array}$ & $\begin{array}{c}\text { TT } \\
\text { amount } \\
(\mathbf{n m o l})\end{array}$ & $\begin{array}{c}\text { TT:GMBS } \\
\text { ratio } \\
(\mathbf{m o l} / \mathbf{m o l})\end{array}$ & $\begin{array}{c}\text { GMBS } \\
(\mathbf{m M})^{\mathbf{b}}\end{array}$ \\
\hline 1 & 7 & 4 & 15 & 13.5 & 96 & 10 & 16 \\
\hline 2 & 8 & 4 & 15 & 13.5 & 96 & 10 & 16 \\
\hline 3 & 7 & 37 & 15 & 13.5 & 96 & 10 & 16 \\
\hline 4 & 8 & 37 & 15 & 13.5 & 96 & 10 & 16 \\
\hline 5 & 7 & 4 & 55 & 49.5 & 352 & 10 & 59 \\
\hline 6 & 8 & 4 & 55 & 49.5 & 352 & 10 & 59 \\
\hline 7 & 7 & 37 & 55 & 49.5 & 352 & 10 & 59 \\
\hline 8 & 8 & 37 & 55 & 49.5 & 352 & 10 & 59 \\
\hline 9 & 7 & 4 & 15 & 13.5 & 96 & 160 & 256 \\
\hline 10 & 8 & 4 & 15 & 13.5 & 96 & 160 & 256 \\
\hline 11 & 7 & 37 & 15 & 13.5 & 96 & 160 & 256 \\
\hline 12 & 8 & 37 & 15 & 13.5 & 96 & 160 & 256 \\
\hline 13 & 7 & 4 & 55 & 49.5 & 352 & 160 & 939 \\
\hline 14 & 8 & 4 & 55 & 49.5 & 352 & 160 & 939 \\
\hline 15 & 7 & 37 & 55 & 49.5 & 352 & 160 & 939 \\
\hline 16 & 8 & 37 & 55 & 49.5 & 352 & 160 & 939 \\
\hline 17 & 7.5 & 20.5 & 35 & 31.5 & 224 & 40 & 149 \\
\hline 18 & 7.5 & 20.5 & 35 & 31.5 & 224 & 40 & 149 \\
\hline 19 & 7.5 & 20.5 & 35 & 31.5 & 224 & 40 & 149 \\
\hline
\end{tabular}

\section{a: initial volume was $300 \mu \mathrm{L}$}

b: initial volume was $20 \mu \mathrm{L}$

\subsubsection{Statistical Analysis DoE Modification}

The statistical analysis as available in the software module used (MODDE, Umetrics), can address several quality attributes to evaluate the quality and output of the model. Distribution of the response factors and the N-residuals plot showed normal profiles (data not shown). For the coefficients plots, all coefficients, which were non-significant for all three response factors, were removed. This yielded specific coefficients, which were applied to all three output parameters to construct similar models. 

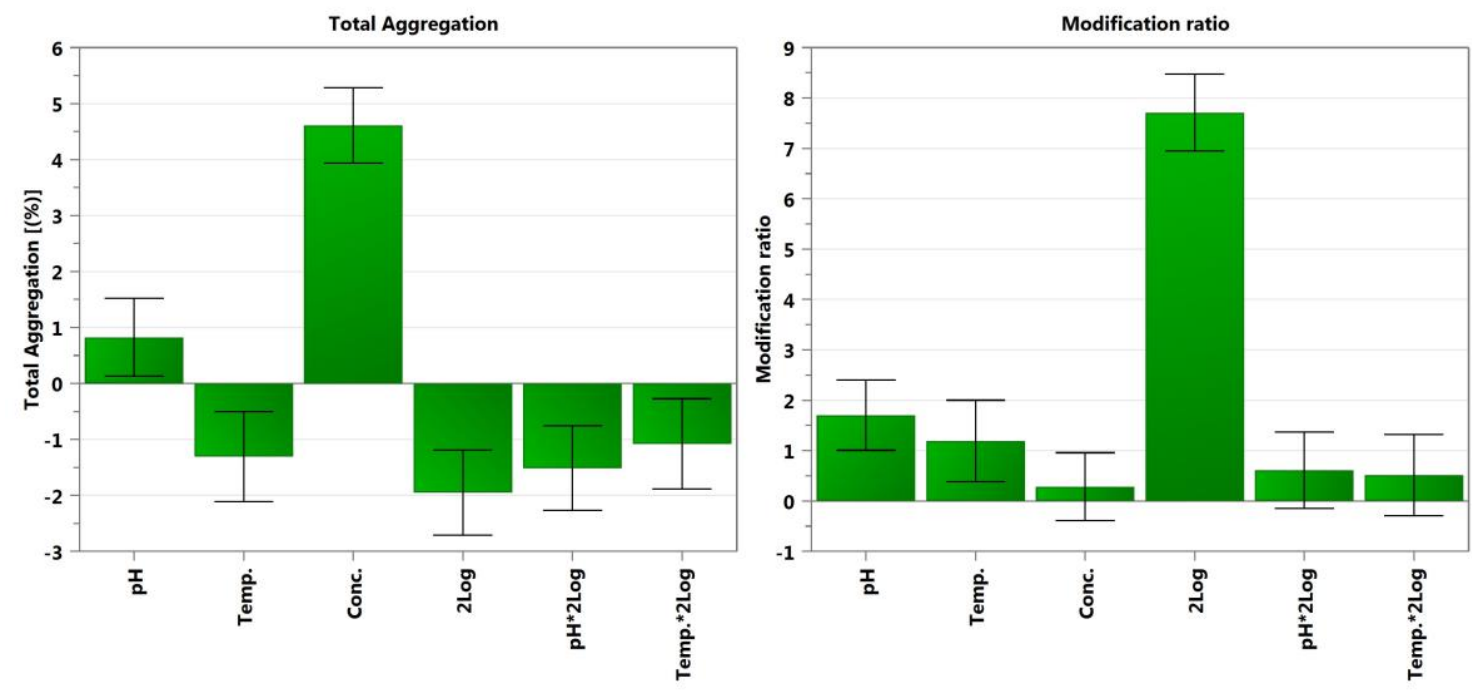

Figure S11: Coefficients plot for modification and aggregation.

\subsubsection{Summary of fit results}

When evaluating the summary of fit plot; the R2, Q2 and reproducibility all met specifications (Table S4). The model validity for the modification ratio is low (- 0.20), which is a direct effect of the extremely high reproducibility (0.99). For this reason and due to the results for the other performance indicators, the model validity for the modification ratio was ignored. As a final representation of the model performance, the observed verses predicted plots all showed acceptable R2 (>0.6) for the model applied and the analysis of variance (ANOVA) analysis showed $\mathrm{p}<0.05$ for the regression and $\mathrm{p}>0.05$ for the lack of fit for all models (data not shown).

Table S4: Summary of fit results

\begin{tabular}{|l|c|c|c|c|}
\hline & $\begin{array}{c}\text { Reference } \\
\text { values }\end{array}$ & $\begin{array}{c}\text { Total } \\
\text { aggregation }\end{array}$ & $\begin{array}{c}\text { Aggregation } \\
\text { increase }\end{array}$ & $\begin{array}{c}\text { Modification } \\
\text { ratio }\end{array}$ \\
\hline $\mathbf{R 2}^{\mathbf{a}}$ & n.a. & 0.80 & 0.71 & 0.88 \\
\hline $\mathbf{Q 2}^{\mathbf{b}}$ & $>0.50$ & 0.75 & 0.62 & 0.86 \\
\hline $\mathbf{R 2}^{-} \mathbf{Q 2}^{\mathbf{c}}$ & $<0.20-0.30$ & 0.05 & 0.09 & 0.02 \\
\hline Model validity $^{\mathbf{d}}$ & $>0.25$ & 0.83 & 0.52 & -0.20 \\
\hline Reproducibility $^{\mathbf{e}}$ & $>0.50$ & 0.77 & 0.76 & 0.99 \\
\hline
\end{tabular}

a: goodness of fit, is a measure of how well the regression model can be made to fit the raw data; b: goodness of prediction, estimates the predictive power of the model; c: for a model to pass this diagnostic test, both R2 and Q2 should be high, and preferably not separated by more than $0.2-0.3$. A larger difference constitutes a warning of an inappropriate model; $d$ : 
reflects whether a model is appropriate in a general sense, whether the right type of model was chosen; e: constitutes the pure error and control of the experimental procedure

\subsubsection{Sweet spot analysis}

When combining the models for total aggregation, aggregation increase and modification ratio, a sweet spot analysis was created (Figure S12). The chosen set points were a minimum modification ratio of 22.0, a maximum of $7.5 \%$ aggregation increase and a maximum of $34.3 \%$ total aggregation. The sweet spot analysis ruled out the higher (35 and $55 \mathrm{mg} / \mathrm{mL}$ ) concentrations of TT and also the lower GMBS:TT ratios (2log $3.32(1: 10)$ and $2 \log 5.32$ (1:40)). When looking at the upper left graph (TT concentration at $15 \mathrm{mg} / \mathrm{mL}$ and GMBS:TT ratios $2 \log 7.32$ ), the sweet spot is shown in green. There is a broad range for both $\mathrm{pH}$ and temperature where the optimal outcome of the modification can be obtained. Sweet spot analysis showed that a process at ambient temperature is feasible for the chosen conditions. This is preferable for ease of the production process design. When applying general temperature conditions $\left(15-25^{\circ} \mathrm{C}\right)$ at $15 \mathrm{mg} / \mathrm{mL}$ and GMBS:TT ratio of $2 \log 7.32$ (1:160), a range of operations for $\mathrm{pH}$ between 7.5 and 8.0 would yield modified TT 4 according to the set specifications.

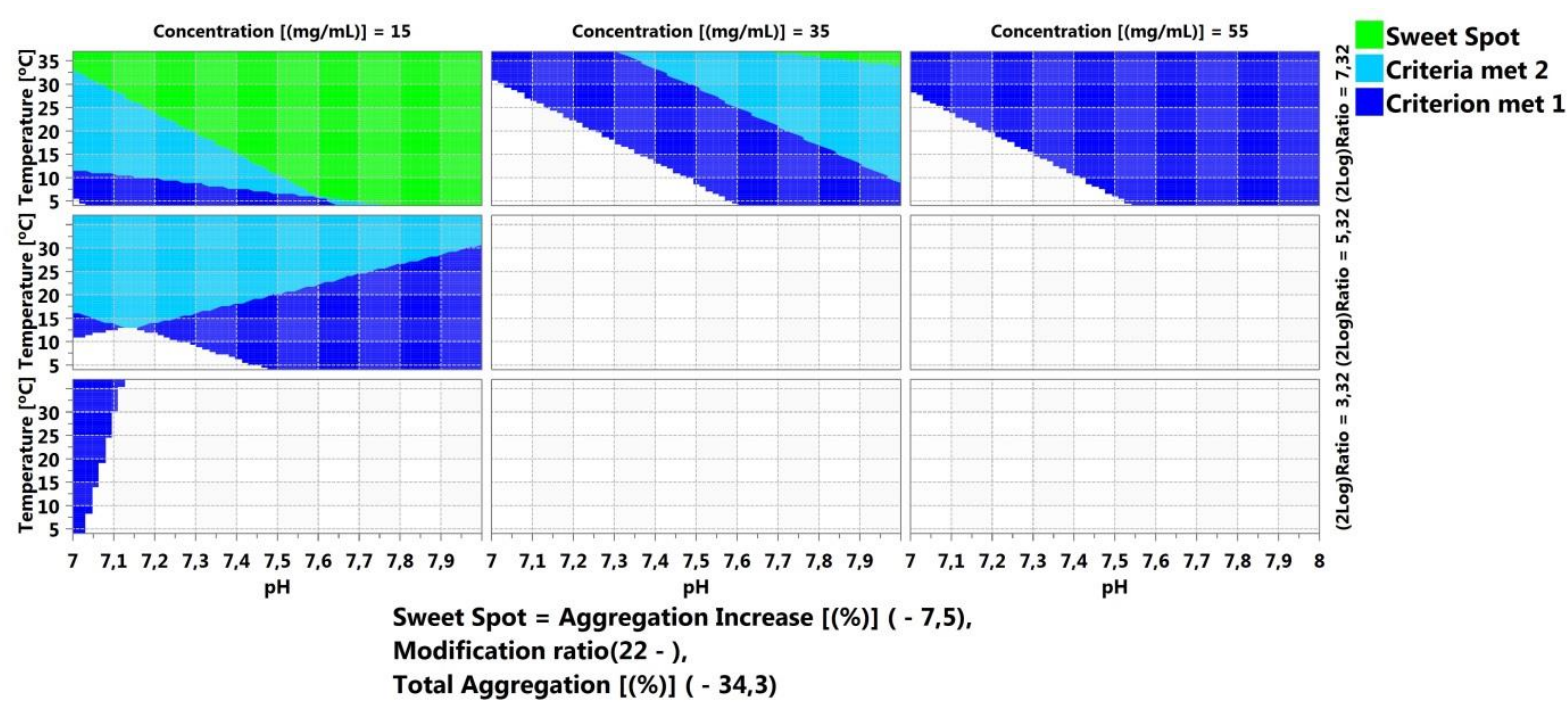

Figure S12: Sweet spot plot analysis 


\subsubsection{Robustness of DoE modification}

A robustness investigation was performed, applying the specifications of the sweet spot at which the modification yields optimal results (Table S5). The same factors as for the screening DoE were investigated, this time with respect to the variation that can be readily expected for the individual factors. For the variation in temperature, it was decided to test for robustness over a broader range, which covered the full range for ambient temperature (15 $\left.25^{\circ} \mathrm{C}\right)$. For the robustness of the DoE, the minimum required response factors as described before would have to apply.

Compared to the DoE for the GMBS-modification, the exact same statistical parameters were reviewed for the quality of the model. All statistical parameters met the specifications. Again, when assessing the summary of fit, the model validity for the modification ratio was too low $(<0.25)$, which was again omitted for reasons described before (data not shown). When putting the models for total aggregation, aggregation increase and modification ratio, together a sweet spot analysis was created. The chosen set points, as described, were that a minimum modification ratio of 22.0 , a maximum of $7.5 \%$ aggregation increase and a maximum of $34.3 \%$ total aggregation would be tolerated (Table S6). The sweet spot analysis showed that even when variations were applied to the different parameters, the outcome of the modification still complied with the set requirements (data not shown).

Table S5: Robustness of DoE modification settings

\begin{tabular}{|c|c|c|c|c|}
\hline Factors & $\begin{array}{c}\text { Expected variation } \\
(\boldsymbol{\%})\end{array}$ & Low & Mid & high \\
\hline TT concentration $(\mathbf{m g} / \mathbf{m L})$ & 10 & 13.5 & 15 & 16.5 \\
\hline 2log (ratio) TT:GMBS & 10 & $7.16(143)$ & $7.32(160)$ & $7.48(178)$ \\
\hline $\mathbf{p H}$ & 2.5 & 7.6 & 7.8 & 8.0 \\
\hline Temperature $\left({ }^{\circ} \mathbf{C}\right)$ & 12 & 15 & 20 & 25 \\
\hline
\end{tabular}

Table S6: Robustness of DoE modification response factors

\begin{tabular}{|c|c|c|}
\hline Responses & Minimum & Maximum \\
\hline Total aggregation (\%) & n.s. $^{\text {a }}$ & 34.3 \\
\hline Aggregation increase (\%) & n.s. $^{\text {a }}$ & 7.5 \\
\hline Mal:TT ratio (modified groups) & 22.0 & n.s. $^{\text {a }}$ \\
\hline
\end{tabular}

a: Not specified 


\subsubsection{Removal of free pentadecasaccharide after conjugation}

After conjugation is completed, the conjugates were purified using buffer exchange. Initial experiments showed that using a $10 \mathrm{kDa}$ MWCO lead to remaining free pentadecasaccharide. However, changing to a $30 \mathrm{kDa}$ MWCO showed complete removal of the free pentadecasaccharide (Figure S13).

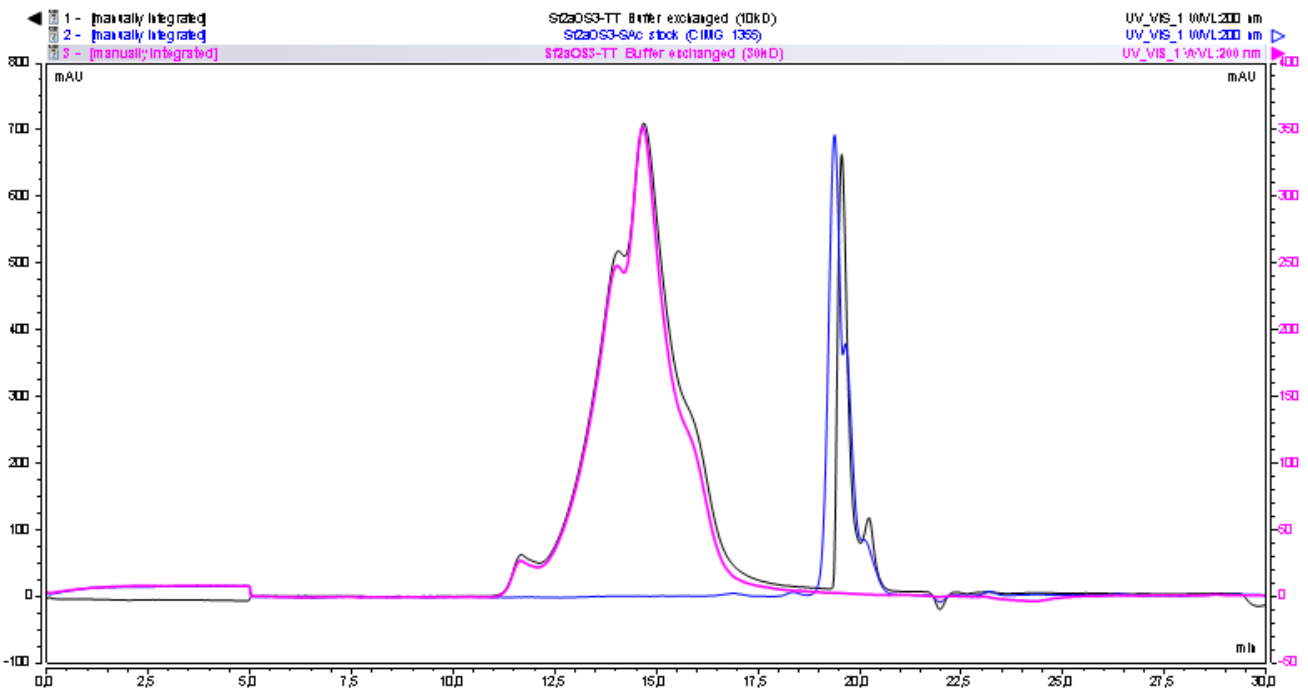

Figure S13: HPLC profiles comparing purification of conjugates using $10 \mathrm{kDa}$ and $30 \mathrm{kDa}$ MWCO filters: Black trace: conjugate processed using a $10 \mathrm{kD}$ cut-off filter with a peak at approximately $19.5 \mathrm{~min}$ representing the remaining forms of the pentadecasaccharide; Blue trace: pentadecasaccharide with characteristic elution at approximately $19.5 \mathrm{~min}$; Pink trace: conjugate processed using a $30 \mathrm{kD}$ cut-off filter 


\section{Immunogenicity analysis}

Statistical analysis: the Mann Whitney test was used for comparing groups of mice.

C1

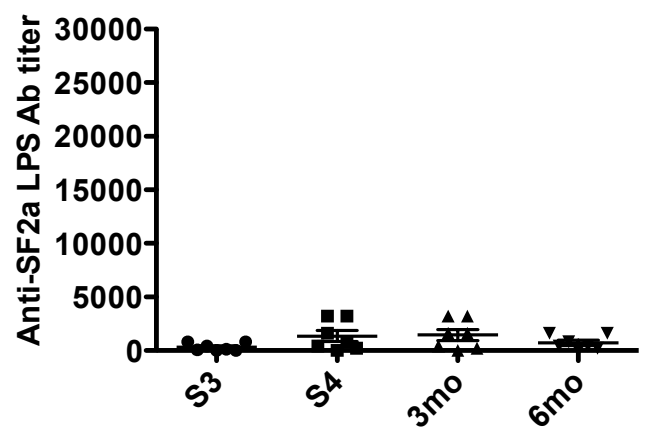

C3

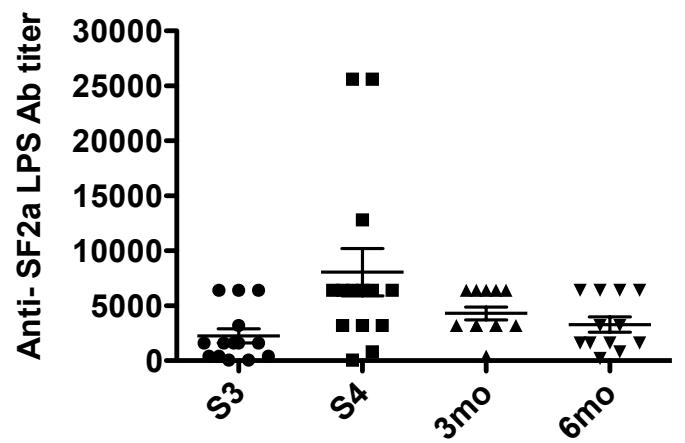

C2

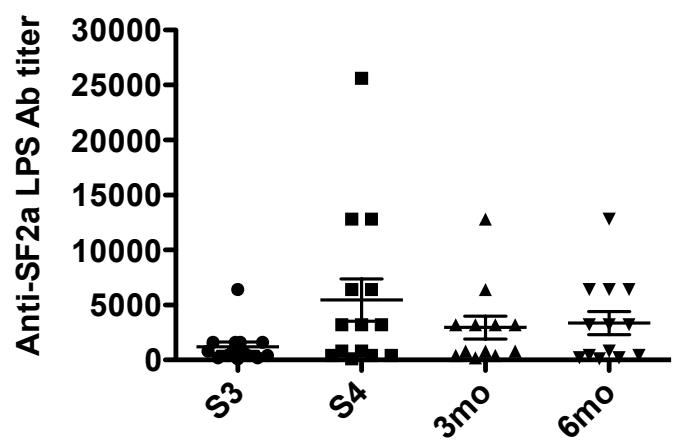

C4

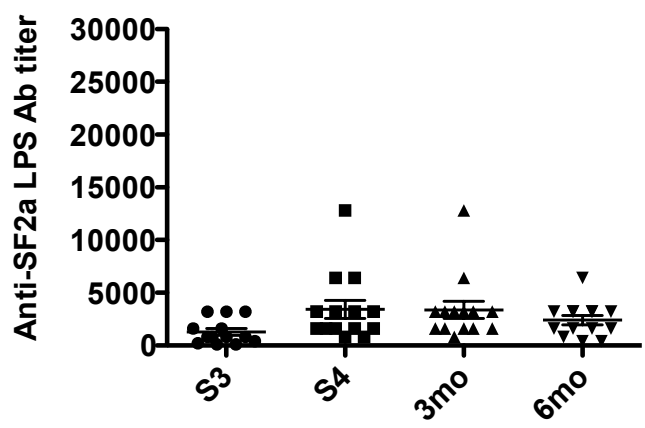

Figure S14: Anti-SF2a LPS IgG titer induced in mice receiving 3 (S3) and 4 (S4) times a dose of glycoconjugates C1-C4 containing $2.5 \mu \mathrm{g}$ of $[\mathbf{A B}(\mathbf{E}) \mathbf{C D}]_{3}$ and sustained response after 3 and 6 months ( $3 \mathrm{mo}$ and $6 \mathrm{mo}$, respectively). 